\title{
Spatially Resolved Pharmacokinetic Rate Images of ICG using Near Infrared Optical Methods
}

\author{
Burak Alacam $^{a}$, Birsen Yazıc1 $^{a},{\text { Xavier } \text { Intes }^{b}, \text { Shoko Nioka }^{c} \text {, and Britton Chance }}^{c}$ \\ ${ }^{a}$ Electrical, Computer and Systems Engineering Dept., Rensselaer Polytechnic Institute, USA \\ ${ }^{b}$ ART Advanced Research Technologies Inc., Canada \\ ${ }^{c}$ Department of Biochemistry and Biophysics, University of Pennsylvania, USA
}

\begin{abstract}
In this work, we present spatially resolved pharmacokinetic rate images of indocyanine green (ICG) obtained from three breast cancer patients using near infrared imaging methods. We used a two-compartment model, namely, plasma and extracellular extravascular (EES), to model ICG kinetics around the tumor region. We introduced extended Kalman filtering (EKF) framework to estimate the ICG pharmacokinetic rate images. The EKF framework allows simultaneous estimation of pharmacokinetic rates and the ICG concentrations in each compartment. Based on the pharmacokinetic rate images, we observed that the rates from inside and outside the tumor region are statistically different with a p-value of 0.0001 for each patient. Additionally, we observed that the ICG concentrations in plasma and the EES compartments are higher around the tumors agreeing with the hypothesis that ICG may act as a diffusible extravascular flow in leaky capillary of cancer vessels. Our study shows that spatially resolved pharmacokinetic rate images can be potentially useful for breast cancer screening and diagnosis.
\end{abstract}

Keywords: extended Kalman filter, indocyanine green, pharmacokinetic analysis, breast cancer.

\section{INTRODUCTION}

Near Infrared (NIR) optical imaging has recently emerged as a potential diagnostic tool for breast cancer. NIR imaging has several advantages over existing imaging modalities. It is easy to use, minimally invasive, relatively inexpensive and can be made portable. More importantly, in conjunction with molecular imaging probes, NIR imaging techniques can provide cellular, molecular level information, which can be used for cancer imaging. In particular, dynamic tomographic imaging methods can produce absorption images which can be used to derive kinetics of molecular probes. The kinetic rates can then be used as potential detection and diagnosis tools.

Presently, ICG is the only FDA approved fluorescent agent for human use. It is hypothesized that ICG may act as a blood flow indicator in tight capillaries of normal vessels and as a diffusible (extravascular) flow in leaky capillary of cancer vessels. ${ }^{1,4}$ A number of studies using compartmental modeling were reported to show the feasibility of using ICG pharmacokinetics in tumor characterization. ${ }^{1-5}$ Parameters related to capillary permeability were used as malignancy indicators. Gurfinkel et. al. ${ }^{3}$ presented in vivo fluorescent, NIR reflectance images of ICG to discriminate spontaneous canine adenocarcinoma from normal mammary tissue using a two compartmental model. It was claimed that the model parameters show no difference in the ICG uptake rates between normal and diseased tissue. Cuccia et al. ${ }^{4}$ presented a study of the dynamics of ICG in an adenocarcinoma rat tumor model. A two-compartment model describing ICG dynamics was used to quantify physiologic parameters related to capillary permeability. It was shown that different tumor types have different capillary permeability rates. Intes et. al. ${ }^{5}$ presented the uptake of ICG by breast tumors using a continuous wave diffuse optical tomography apparatus. A two-compartment model was used to analyze the pharmacokinetics of ICG. It was shown that the malignant cases exhibit slower rate constants (uptake and outflow) compared to healthy tissue.

The studies above demonstrate the feasibility of the ICG pharmacokinetics in tumor characterization. However, estimation of pharmacokinetic parameters is a challenging problem, because it is a highly non-linear estimation problem with sparse data available in clinical setting. Furthermore, parameter values show variation from

Further author information: Send correspondence to Birsen Yazici, e-mail: yazici@ecse.rpi.edu 
one subject to another. To address these issues, we recently introduced a systematic and robust approach to model, estimate and analyze the ICG pharmacokinetics based on extended Kalman filtering framework. ${ }^{1,2}$ We introduced Bayesian Information Criteria to choose best statistical model fit. EKF based approach can be also utilized to estimate time-dependent pharmacokinetic rates. We tested three different compartmental models for ICG pharmacokinetics using NIR data collected from Fischer rats with cancerous tumors. Our study showed that the permeability rates out of the vasculature are higher in edematous tumors as compared to necrotic tumors.

In this paper, our objective is to obtain spatially resolved pharmacokinetic rate images of ICG, using NIR data acquired from three breast cancer patients. We first develop a set of temporally resolved 2-D ICG concentration images based on linearized diffusion equation. We then estimate the ICG pharmacokinetic rates and the concentrations in each compartment for each pixel based on the EKF framework for the two-compartment model. Reconstructed 2-D pharmacokinetic rate images of the three patient show that the rates from inside and outside the tumor region are statistically different with a p-value of 0.0001. Additionally, the ICG concentrations in plasma and the EES compartments are higher around the tumor agreeing with the hypothesis that around the tumor region ICG may act as a diffusible extravascular flow in leaky capillary of cancer vessels.

The rest of the paper is organized as follows: In Section II, we present the reconstruction of differential absorption and ICG concentration images. In Section III, we present the two-compartment model for ICG. In Section IV, we present the EKF based estimation of ICG pharmacokinetic parameters. In Section V, we present the clinical results obtained from breast cancer patients. Finally, Section VI summarizes our results and conclusion.

\section{DIFFUSE OPTICAL TOMOGRAPHY (DOT) USING ICG}

\subsection{Reconstruction of Bulk ICG Concentration Images}

The propagation of light in soft tissue is modeled by diffusion approximation. ${ }^{6}$ In the forward model, the analytical solutions of the heterogonous diffusion equation given in ${ }^{5}$ is derived using first order Rytov approximation. The sample volume is divided into a set of voxels and the measurements are related to the relative absorption coefficients of each voxel by a system of linear equations. The shape of the breast was approximated as a cylinder and the Kirchhoff approximation ${ }^{7,8}$ for diffuse waves was used to model the interaction of light with boundaries. In order to account for the biological noise, the forward model was implemented with coupling coefficient technique. $^{9}$ The inverse problem was addressed by using singular value decomposition of the MoorePenrose generalized system. Tikhinov regularization was used to stabilize the inversion procedure. The L-curve method was applied to an experimental model reconstruction and the best regularization parameter was derived using a curvature function which was employed in. ${ }^{10}$ A detailed discussion of the forward and inverse models used for the reconstruction of differential absorption coefficients can be found in. ${ }^{5}$

We used the linear relationship between the differential absorption coefficients and concentrations given by the following equation to reconstruct a set of temporally resolved 2-D ICG concentration images. ${ }^{11}$

$$
\Delta \mu_{a}=e C \ln 10=2.303 \text { e } C
$$

where $\Delta \mu_{a}$ denotes the differential absorption coefficient, and $C$ denotes the concentration of ICG, and $e$ is the extinction coefficient of ICG at wavelength $805 \mathrm{~nm}$. Using the linear relationship above, we get 2-D images of ICG concentrations in tissue from differential absorption images reconstructed at different time instants on a pixel by pixel basis. In the following Sections, we will discuss the two-compartment model, and the estimation of pharmacokinetic rates.

\section{ICG PHARMACOKINETICS AND THE TWO-COMPARTMENT MODELING}

\subsection{Compartmental Analysis of ICG Pharmacokinetics}

In this paper, we assume that the tumor region is composed of two compartments; namely plasma and the EES. ${ }^{1,4}$ Figure 1 shows the two-compartment model for ICG kinetics. $C_{p}$ and $C_{e}$ represent the ICG concentrations in plasma and the EES, respectively. The parameters $k_{i n}$ and $k_{\text {out }}$ govern the leakage into and the drainage out 


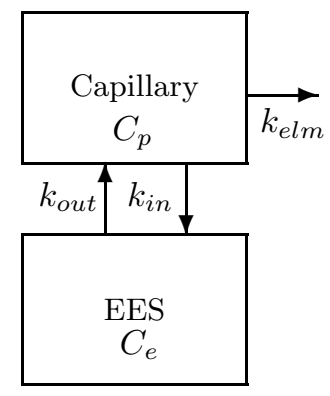

Figure 1. Block diagram of the two-compartment model for ICG pharmacokinetics.

of the EES. The parameter $k_{\text {elm }}$ describes the ICG elimination from the body through kidneys and livers. The parameters $v_{p}$ and $v_{e}$ are plasma and the EES volume fractions, respectively.

The leakage into and the drainage out of the EES is given by:

$$
\frac{d C_{e}(t)}{d t}=-k_{\text {out }} C_{e}(t)+k_{i n} C_{p}(t)
$$

The leakage into and the drainage out of plasma is given by:

$$
\frac{d C_{p}(t)}{d t}=-\left(k_{\text {in }}+k_{\text {elm }}\right) C_{p}(t)+k_{\text {out }} C_{e}(t)
$$

The parameters $k_{i n}, k_{\text {out }}$, and $k_{\text {elm }}$ have a unit of $\mathrm{sec}^{-1}$. They are defined as the permeability surface area products given by $P S \rho$, where $P$ is the capillary permeability constant, $S$ is the capillary surface area, and $\rho$ is the tissue density. We consider transcapillary leakage to occur only at the tumor site.

Actual bulk ICG concentration in the tissue measured by NIR spectroscopy is a linear combination of plasma and the EES ICG concentrations:

$$
m(t)=v_{p} C_{p}(t)+v_{e} C_{e}(t),
$$

where $v_{p}, v_{e}, C_{e}$, and $C_{p}$ are as defined above, and $m(t)$ denotes the bulk ICG concentration.

Here, we consider transcapillary leakage to occur only at the tumor site. We also assume that a small perturbation of the global plasma concentration does not affect the bulk removal.

\section{EXTENDED KALMAN FILTERING FOR THE ESTIMATION OF ICG PHARMACOKINETICS}

Coupled differential equations resulting from the two-compartment model of the ICG pharmacokinetics can be expressed in state-space representation as follows:

$$
\begin{gathered}
{\left[\begin{array}{c}
\dot{C}_{e}(t) \\
\dot{C}_{p}(t)
\end{array}\right]=\left[\begin{array}{cc}
-k_{\text {out }} & k_{\text {in }} \\
k_{\text {out }} & -\left(k_{\text {in }}+k_{\text {elm }}\right)
\end{array}\right]\left[\begin{array}{l}
C_{e}(t) \\
C_{p}(t)
\end{array}\right]+\boldsymbol{\omega}(t),} \\
m(t)=\left[\begin{array}{ll}
v_{e} & v_{p}
\end{array}\right]\left[\begin{array}{c}
C_{e}(t) \\
C_{p}(t)
\end{array}\right]+\eta(t)
\end{gathered}
$$

where $\boldsymbol{\omega}(t)$ and $\eta(t)$ are uncorrelated zero mean Gaussian processes with covariance matrix $\mathbf{Q}$, and variance $\boldsymbol{\sigma}^{2}$, respectively.

In matrix-vector notation, the state-space representation is given by:

$$
d \mathbf{C}(t)=\mathbf{K}(\boldsymbol{\alpha}) \mathbf{C}(t) d t+\boldsymbol{\omega}(t) d t,
$$




$$
m(t)=\mathbf{V}(\boldsymbol{\alpha}) \mathbf{C}(t)+\eta(t) .
$$

where $\mathbf{C}(t)$ denotes the concentration vector with elements $C_{e}(t)$, and $C_{p}(t) ; \mathbf{K}(\boldsymbol{\alpha})$ is the $2 \times 2$ system matrix, $\mathbf{V}(\boldsymbol{\alpha})$ is the $1 \times 2$ measurement matrix as defined in equation (5) and $\boldsymbol{\alpha}$ is the parameter vector given by

$$
\boldsymbol{\alpha}=\left[\begin{array}{lllll}
k_{\text {out }} & k_{\text {in }} & k_{\text {elm }} & v_{e} & v_{p}
\end{array}\right] \text {. }
$$

The ICG measurements in equation (6) are collected at discrete time instances, $t=k T, k=0,1, \ldots$, where $T$ is the sampling period. Therefore, the continuous model given in Equation (6) has to be discretized. To simplify our notation, we shall use $\mathbf{C}(k)=\mathbf{C}(k T)$ and $m(k)=m(k T)$. The corresponding discrete state-space model is given by:

$$
\begin{gathered}
\mathbf{C}(k+1)=\mathbf{K}_{d}(\boldsymbol{\alpha}) \mathbf{C}(k)+\boldsymbol{\omega}(k) \\
m(k)=\mathbf{V}_{d}(\boldsymbol{\alpha}) \mathbf{C}(k)+\eta(k),
\end{gathered}
$$

where $\mathbf{K}_{d}(\boldsymbol{\alpha})=e^{\mathbf{K}(\boldsymbol{\alpha})}$ is the discrete time system matrix and $\mathbf{V}_{d}(\boldsymbol{\alpha})=\mathbf{V}(\boldsymbol{\alpha})$ is the discrete measurement matrix. $\boldsymbol{\omega}(k)$ and $\eta(k)$ are zero mean Gaussian white noise processes with covariances matrix $\mathbf{Q}_{d}$ and variance $\boldsymbol{\sigma}_{d}^{2}$, respectively.

An explicit form for the discrete time state-space model is given as follows:

$$
\begin{gathered}
{\left[\begin{array}{c}
C_{e}(k+1) \\
C_{p}(k+1)
\end{array}\right]=\left[\begin{array}{ll}
\tau_{11} & \tau_{12} \\
\tau_{21} & \tau_{22}
\end{array}\right]\left[\begin{array}{l}
C_{e}(k) \\
C_{p}(k)
\end{array}\right]+\boldsymbol{\omega}(k)} \\
m(k)=\left[\begin{array}{ll}
v_{e} & v_{p}
\end{array}\right]\left[\begin{array}{l}
C_{e}(k) \\
C_{p}(k)
\end{array}\right]+\eta(k),
\end{gathered}
$$

where $\tau_{i j}$ is the $i^{\text {th }}$ row and $j^{\text {th }}$ column entry of the system matrix $\mathbf{K}_{d}(\boldsymbol{\alpha})$. The matrix entry $\tau_{i j}$ is an exponential function of the parameters $k_{i n}, k_{\text {out }}$ and $k_{e l m}$. To simplify the estimation process, we shall first estimate $\tau_{i j}$ 's and then compute the pharmacokinetic parameters $k_{i n}, k_{\text {out }}$ and $k_{\text {elm }}$.

We use the EKF algorithm for the joint estimation of the unknown pharmacokinetic parameters, $k_{\text {in }}, k_{\text {out }}$, and $k_{e l m}$, volume fractions, $v_{e}$, and $v_{p}$, and ICG concentrations in plasma and the EES, $C_{p}$, and $C_{e}$. In a linear state-space model when both states (ICG concentrations) and system parameters (pharmacokinetic rates and volume fractions) are unknown, the linear state-space model can be regarded as a non-linear model in which the linear system parameters and states are combined to form the new states of the non-linear model. This system is then linearized and solved for the new unknown states using the EKF estimator. A detailed discussion of the Kalman filtering algorithm for joint estimation of the pharmacokinetic parameters and ICG concentrations in different compartments can be found in. ${ }^{1,2}$

\section{CLINICAL RESULTS}

\subsection{Apparatus}

In this work, we used the data collected with a continuous wave (CW) NIR imaging apparatus. The apparatus has 16 light sources, which are tungsten bulbs with less than 1 watt of output energy. They are located on a circular holder at an equal distance from each other with 22.5 degree apart. Sixteen detectors, namely, silicon photodiodes, are situated in the same plane. The breast is arranged in a pendular geometry with the sourcedetector probes gently touching its surface. Figure 2 illustrates the configuration of the apparatus and the configuration of the detectors and the sources in a circular plane. A band pass filter at $805 \mathrm{~nm}$, the absorption peak of ICG, is placed in front of the sources to select the desired wavelength. A set of data for one source is collected every $500 \mathrm{~ms}$. The total time for a whole scan of the breast including 16 sources and 16 detectors is 8.8 seconds. The detectors use the same positions as the sources to collect the light originating from one source at a time. Only the signals from the farthest 11 detectors are used in the analysis. For example, when Source 1 is on, the data is collected using detectors 4 to 14. A more detailed explanation of the apparatus and the data collection procedure can be found in. ${ }^{12}$ 

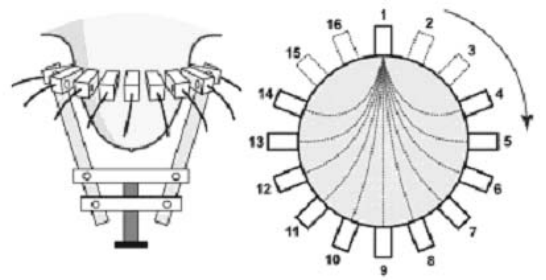

Figure 2. The cut section of the 16 light source-detector device, holding a human breast inside. The diameter can be fitted easily. The 16 light source-detector combinations in each arm are located equal distance, but when the device fits the breast, only the diameter chances.

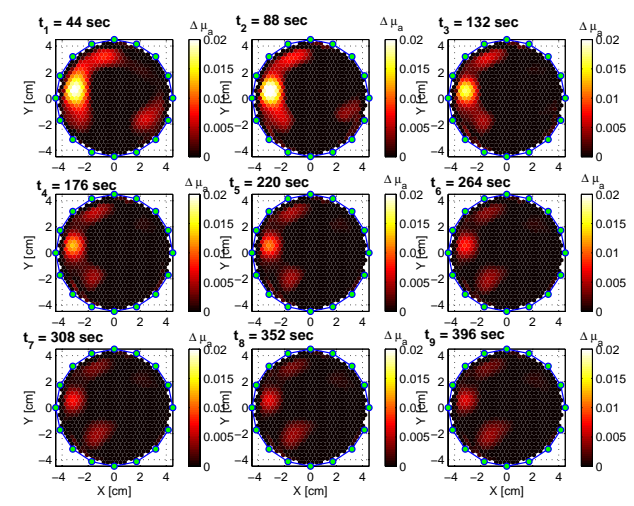

Figure 3. Differential absorption reconstruction images for a set of time instants for Case 1.

\subsection{Protocol}

Patients with suspicious breast tumors were enrolled for this study. ICG was injected intravenously by bolus with a concentration of $0.25 \mathrm{mg}$ per $\mathrm{kg}$ of body weight. Diagnostic information is obtained using biopsy results. Since biopsy modifies the blood volume and blood flow around the tumor region, measurements were made before the biopsy. Data acquisition started before the injection of ICG and continued for 10 minutes.

\subsection{Tumor Information}

Three different patients with different tumor types are included in this study. First case, Case 1, is fibroadenoma, which corresponds to a mass estimated to be $1-2 \mathrm{~cm}$ in diameter within a breast of $9 \mathrm{~cm}$ diameter. Second case, Case 2, is adenocarcinoma corresponding to a tumor estimated to be $2-3 \mathrm{~cm}$ in diameter within a breast of 7.7 $\mathrm{cm}$ diameter. The third case, Case 3 , is invasive ductal carcinoma, which corresponds to a mass estimated to be $3-4 \mathrm{~cm}$ in diameter.

\subsection{ICG Concentration Measurements for Pharmacokinetic Parameter Estimations}

Using the CW imager described above, for each patient, sufficient number of source detector readings were collected from different angles. Reconstruction of differential absorption images using the source-detector readings was discussed in Intes et al.. ${ }^{5}$ A sample set of reconstructed differential absorption images for Case 1, Case 2, and Case 3 for 9 selected time instants are shown in Figure 3, 4, and 5, respectively. Although only 9 reconstructions are displayed, there are approximately 50 reconstructions, each corresponding to a different time instant for each patient. Here, each 2-D image is composed of 649 pixels.

Using the linear relationship between ICG concentration and absorption coefficient, we obtained ICG concentration images from differential absorption images for each case. A sample set of ICG concentration images for the selected time instants is shown in Figure 6,7, and 8 for Cases 1,2 and 3, respectively. Here, the concentration images represent bulk ICG concentration in the tissue, not specifically in plasma or the EES. 


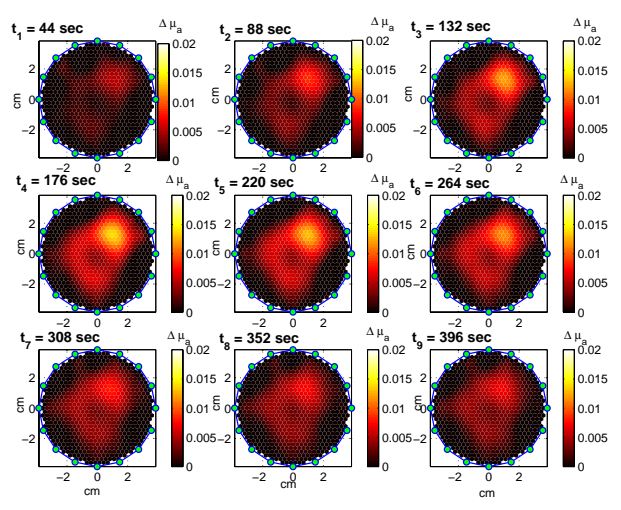

Figure 4. Differential absorption reconstruction images for a set of time instants for Case 2.

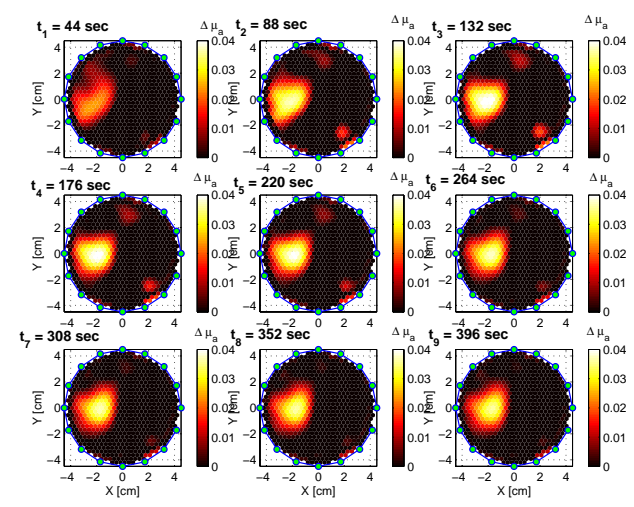

Figure 5. Differential absorption reconstruction images for a set of time instants for Case 3.

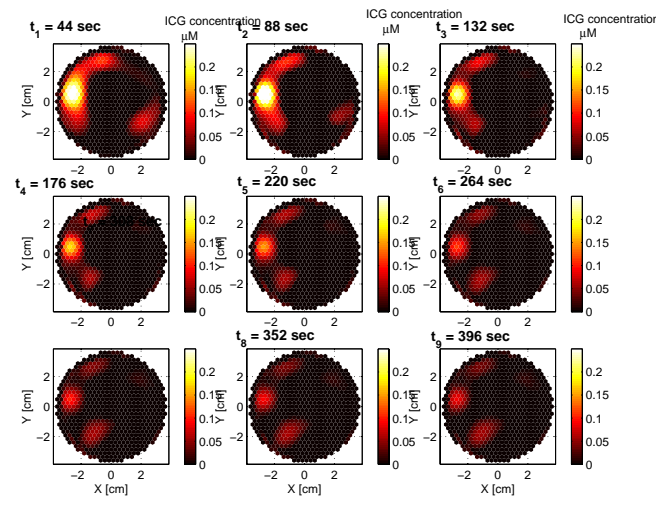

Figure 6. 2-D ICG concentration images for a set of time instants for Case 1. 


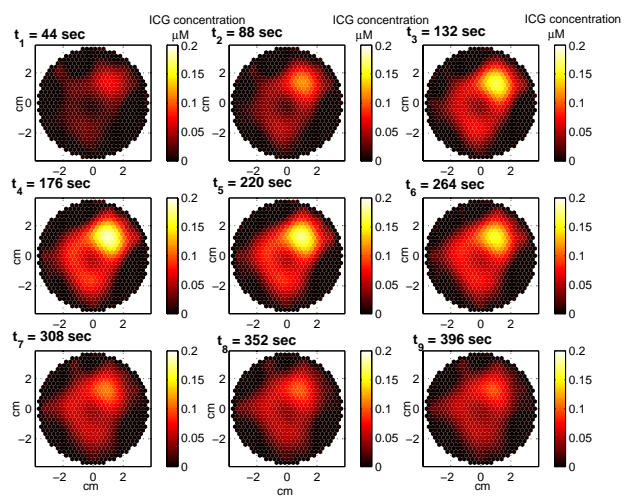

Figure 7. 2-D ICG concentration images for a set of time instants for Case 2.

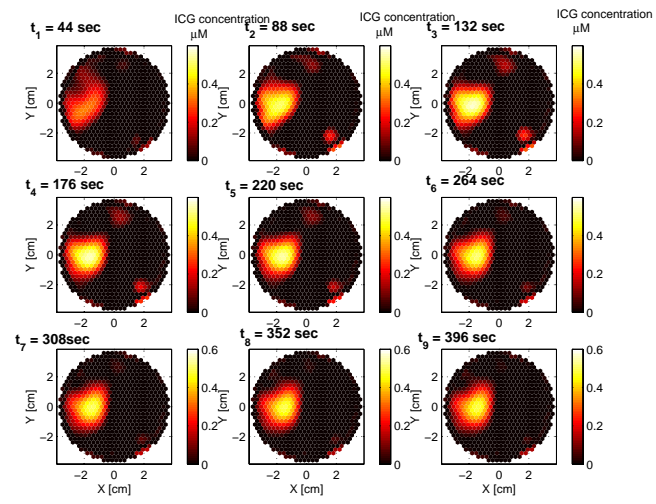

Figure 8. 2-D ICG concentration images for a set of time instants for Case 3.

Using the ICG concentration curves, we estimated the pharmacokinetic parameters for each pixel based on the two-compartment model. We then constructed 2-D permeability rate images using values of these parameters. 2-D images for $k_{\text {in }}, k_{\text {out }}$ for two-compartmental model for each case are shown in Figures 9 (a), (b), 10 (a), (b), and 11 (a), (b), respectively. We also analyzed whether the kinetic rates are statistically different or not for the inside and outside the tumor region. The $k_{i n}$ and $k_{\text {out }}$ values from inside and outside the tumor region are statistically different with a p-value of less than 0.0001 for all cases.

We constructed 2-D ICG concentration images for plasma and the EES. Figures 12-17 show the ICG concentration in the EES and plasma for 3 different time instants for Case 1, 2, 3, respectively. We observed that ICG concentrations in plasma and the EES compartments are higher around the tumors agreeing with the hypothesis that around tumor region ICG may act as a diffusible extravascular flow in leaky capillary of cancer vessels.

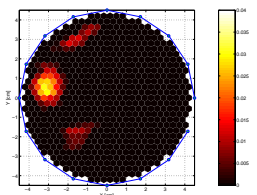

(a)

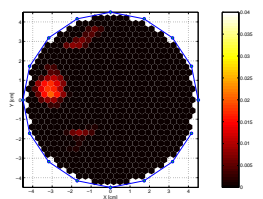

(b)

Figure 9. 2-D images for pharmacokinetic rates $k_{\text {in }}$ and $k_{\text {out }}$ for Case 1. 


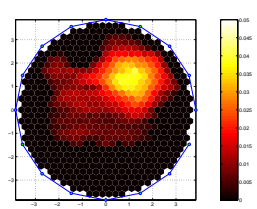

(a)

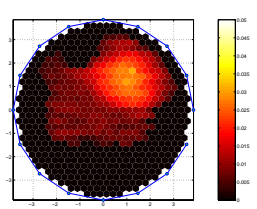

(b)

Figure 10. 2-D images for pharmacokinetic rates $k_{i n}$ and $k_{\text {out }}$ for Case 2.

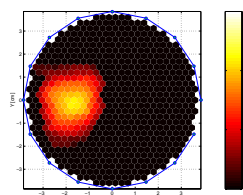

(a)

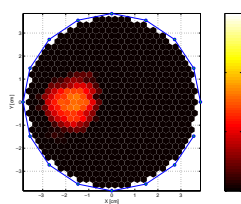

(b)

Figure 11. 2-D images for pharmacokinetic rates $k_{\text {in }}$ and $k_{\text {out }}$ for Case 3.

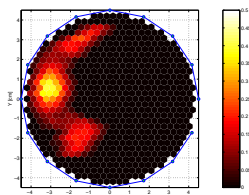

(a)

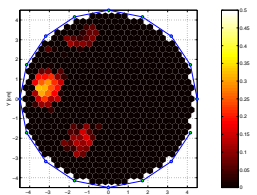

(b)

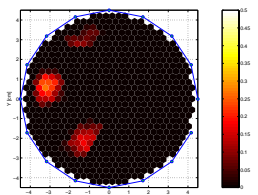

(c)

Figure 12. 2-D ICG concentration images in plasma for Case 1 for the $246.4^{\text {th }}, 334.4^{\text {th }}$, and $422.4^{\text {th }}$ seconds.

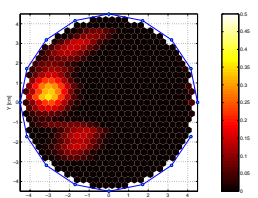

(a)

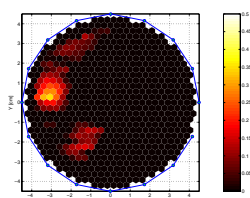

(b)

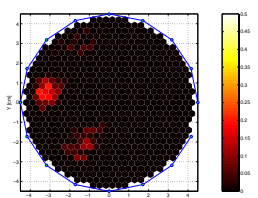

(c)

Figure 13. 2-D ICG concentration images in the EES for Case 1 for the $246.4^{\text {th }}, 334.4^{\text {th }}$, and $422.4^{\text {th }}$ seconds.

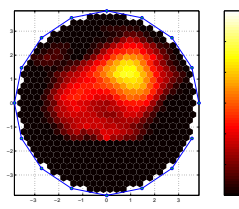

(a)

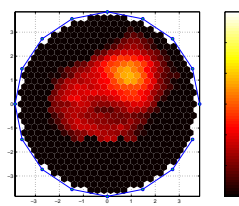

(b)

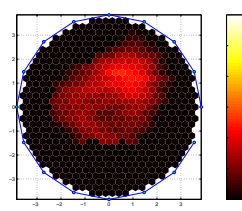

(c)

Figure 14. 2-D ICG concentration images in plasma for Case 2 for the $228.8^{\text {th }}, 316.8^{\text {th }}$, and $404.8^{\text {th }}$ seconds.

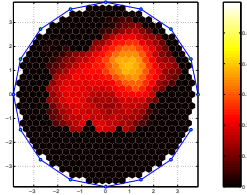

(a)

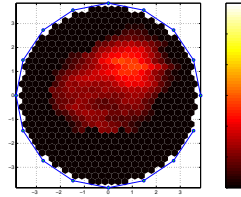

(b)

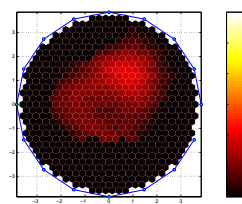

(c)

Figure 15. 2-D ICG concentration images in the EES for Case 2 for the $228.8^{t h}, 316.8^{t h}$, and $404.8^{t h}$ seconds. 


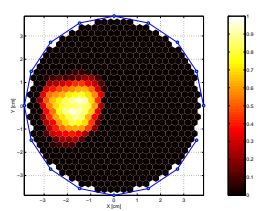

(a)

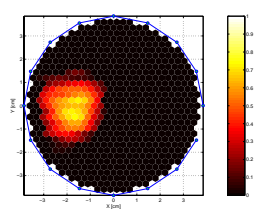

(b)

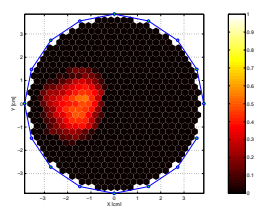

(c)

Figure 16. 2-D ICG concentration images in plasma for Case 3 for the $246.4^{t h}, 378.4^{t h}$, and $510.4^{\text {th }}$ seconds.

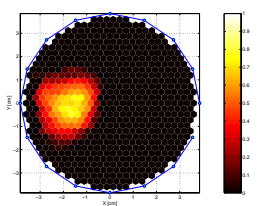

(a)

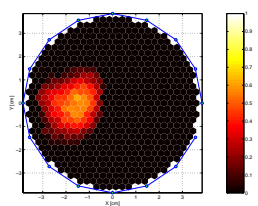

(b)

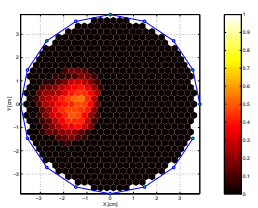

(c)

Figure 17. 2-D ICG concentration images in the EES for Case 3 for the $246.4^{\text {th }}, 378.4^{\text {th }}$, and $510.4^{\text {th }}$ seconds.

\section{CONCLUSION}

In this study, we presented spatially resolved ICG pharmacokinetic images together with the ICG concentrations in plasma and the EES using NIR data obtained from three breast cancer patients. We observed that the pharmacokinetic rates are higher inside the tumor region compared to the outer region; and the ICG concentrations in plasma and the EES are higher around the tumor region. Our study indicates that pharmacokinetic images can be potentially useful to characterize tumor metabolism and angiogenesis. In the near future, we plan to model gadolinium kinetics and compare with ICG kinetics and biopsy findings.

\section{REFERENCES}

1. B. Alacam, B. Yazici, X. Intes, B. Chance "Extended Kalman Filtering Framework for the Modeling and Analysis of ICG Pharmacokinetics," Proc. Of 2005 SPIE Photonic West, San Jose, California USA, 22 - 27 January 2005, vol. 5693. pp. 17-27.

2. B. Alacam, B. Yazici, X. Intes, B. Chance "Analysis of ICG Pharmacokinetics in Cancerous Tumors using NIR Optical Methods," Proc. of EMBS - 27 th Anniversary Conference, Shanghai, China, September 2005.

3. M. Gurfinkel, A. B. Thompson, W. Ralston, T. L. Troy, A. L. Moore, T. A. Moore, J. D. Gust, D. Tatman, J. S. Reynolds, B. Muggenburg, K. Nikula, R. Pandey, R. H. Mayer, D. J. Hawrysz, and E. M. Sevick-Muraca, "Pharmacokinetics of ICG and HPPH-car for the detection of normal and tumor tissue using fluorescence, near-infrared reflectance imaging: a case study," Photochem. Photobiol., Vol. 72, 2000, pp. 94-102.

4. D.J. Cuccia, F. Bevilacqua, A. J. Durkin, S. Merritt, B. J. Tromberg, G. Gulsen, H. Yu, J. Wang, and O. Nalcioglu, "In vivo quantification of optical contrast agent dynamics in rat tumors by use of diffuse optical spectroscopy with magnetic resonance imaging coregistration," Applied Optics, Vol. 42, No 1, June 2003.

5. X. Intes, J. Ripoll, Y. Chen, S. Nioka, A. G. Yodh, B. Chance, "In vivocontinuous-wave optical breast imaging enhanced with Indocyanine Green," Med. Phys. Vol. 30-6, June 2003.

6. A. Yodh and B. Chance, "Spectroscopy and imaging with diffusing light," Phys. Today 48(3), 3440, 1995.

7. J. Ripoll, V. Ntziachristos, R. Carminati, and M. Nieto-Vesperinas, "Kirchhoff approximation for diffusive waves," Phys. Rev. E 64, 051917, 2001.

8. J. Ripoll, V. Ntziachristos, J. P. Culver, D. N. Pattanayak, A. G. Yodh, and M. Nieto-Vesperinas, "Recovery of optical parameters in multiplelayered diffusive media: theory and experiments," J. Opt. Soc. Am. A 18, $821830,2001$.

9. D. Boas, T. Gaudette, and S. Arridge, "Simultaneous imaging and optode calibration with diffuse optical tomography," Opt. Express 8, 263270, 2001.

10. P. Hansen and D. OLeary, "The use of the L-curve in the regularization of discrete ill-posed problems," SIAM J. Sci. Comput., 14, 1487 1503, 1993. 
11. M. L. J. Landsman, G. Kwant, G. A. Mook, W. G. Zijlstra, "Light- absorbing properties, stability, and spectral stabilization of indocyanine green," J. Appl. Physiol., 40, 575-583, 1976.

12. S. Nioka, Y. Yung, M. Schnall, S. Zhao, S. Orel, C. Xie, B. Chance, and S. Solin, "Optical imaging of breast tumor by means of continuous waves," Adv. Exp. Med. Biol., 411, 227232, 1997. 